\title{
Gravitational-wave data analysis using binary black-hole waveforms
}

\author{
P Ajith \\ Max-Planck-Institut für Gravitationsphysik (Albert-Einstein-Institut) and Leibniz Universität \\ Hannover, Callinstr. 38, 30167 Hannover, Germany
}

Received 3 December 2007, in final form 10 March 2008

Published 15 May 2008

Online at stacks.iop.org/CQG/25/114033

\begin{abstract}
While the inspiral and ring-down stages of the binary black-hole coalescence are well modelled by analytical approximation methods in general relativity, the recent progress in numerical relativity has enabled us to compute accurate waveforms from the merger stage also. This has an important impact on the search for gravitational waves from binary black holes. 'Complete' binary black-hole waveforms can now be produced by matching post-Newtonian waveforms with those computed by numerical relativity, which can be parametrized to produce analytical waveform templates. The 'complete' waveforms can also be used to estimate the efficiency of different search methods aiming to detect signals from black-hole coalescences. This paper summarizes some recent efforts in this direction.
\end{abstract}

PACS numbers: 04.25.D, 04.30.-w, 04.25.dg, 29.85.Fj

(Some figures in this article are in colour only in the electronic version)

\section{Introduction}

Coalescing black-hole binaries are among the most promising sources of gravitational waves for ground-based detectors like LIGO and Virgo, and the planned space-borne detector LISA. The evolution of binary black holes is conventionally split into three stages: inspiral, merger and ring down. In the inspiral stage, the two compact objects, driven by radiation reaction, move in quasi-circular orbits. Eventually approaching the ultra-relativistic regime, the two bodies merge to form a single excited Kerr black hole. In the ring-down stage, the excited black hole loses its energy by gravitational-wave emission and settles into a Kerr black hole. Gravitational waveforms from the inspiral and ring-down stages can be accurately computed by approximation/perturbation techniques in general relativity [1-3]. The recent progress in numerical relativity [4-6] has enabled us to model also the non-perturbative merger phase of the coalescence of binary black holes [7-17]. 
While the current gravitational-wave searches look for each stage of the binary black-hole coalescence separately (see, for example, [18, 19]), combining the results from analytical and numerical relativity enables us to coherently search for all the three stages using a single template family. This coherent search is significantly more sensitive than the current searches over certain mass ranges (see section 4). This search has added advantages: including all the three stages adds more 'structure' to the template waveform, resulting in a potential reduction of false alarms. The additional structure and the improved signal-to-noise ratio also results in an improved estimation of the parameters of the binary, which is particularly important for LISA data analysis. As LISA data will contain a 'cocktail' of many strong binary signals, these will have to be subtracted from the data in order to analyse other signals. Improved parameter estimation can also have a tremendous impact in cosmology. Since many of the supermassive black-hole mergers are likely to have electromagnetic counterparts, it is possible to constrain the values of cosmological parameters by combining the gravitational-wave and electromagnetic observations [20]. In particular, using the distance-redshift relation from many binary black-hole 'standard sirens', LISA might be able to put interesting constraints on the equation of state of the dark energy [21]. The error bars on this depend on how accurately the 'red-shifted' mass of the source and the luminosity distance are estimated, and how well the host galaxy of the electromagnetic counterpart is identified. The improved parameter estimation might help to tighten these constraints.

Several authors have proposed different ways of computing gravitational-wave templates containing all the three stages of the binary black-hole coalescence [22-26]. In particular, [25] proposed a phenomenological parametrization for non-spinning binary black-hole waveforms. These waveforms are explicit functions of the physical parameters of the system and exhibited very high overlaps with the 'target signals'. The target signals were constructed by matching numerical-relativity waveforms with post-Newtonian waveforms in appropriate matching regions. These waveforms contain all the three stages of the binary black-hole coalescence. Hence, they can also be used to estimate the efficiency of different (template-based and other) search methods used to detect signals from binary black-hole coalescences.

This paper provides an overview of the application of the results from analytical and numerical calculations of binary black-hole waveforms into gravitational-wave data analysis. Section 2 describes how 'complete' binary black-hole coalescence waveforms (so-called hybrid waveforms) from non-spinning binaries can be constructed by matching post-Newtonian and numerical-relativity waveforms. Here we also investigate the robustness of the matching procedure by studying the mismatch between hybrid waveforms constructed using different matching regions. Section 3 introduces an analytical two-parameter family of non-spinning waveforms having very good overlaps with the hybrid waveforms. Section 4 shows how the hybrid waveforms can be used to estimate the sensitivity of different searches. Here we compare the fitting factor and faithfulness of different template-based searches using the hybrid waveforms as target signals. Finally, section 5 provides a summary and future plans.

\section{Constructing 'complete' binary black-hole coalescence signals}

Although numerical relativity (NR) is able to compute gravitational waveforms containing all the three stages of the binary black-hole coalescence, the numerical simulations are heavily limited by the computational resources. But, the post-Newtonian (PN) formalism is known to work very well in the early inspiral. Thus, complete binary black-hole coalescence waveforms (hybrid waveforms) can be constructed by matching PN and NR waveforms in an appropriate 
matching region. Different authors have studied the consistency of the non-spinning PN waveforms with NR waveforms. See [24-31] for some of the recent work.

The time-domain waveform $h_{+, \times}(t, \boldsymbol{\mu})$ from a particular system is parametrized by a set of 'extrinsic parameters' $\boldsymbol{\mu}=\left\{\varphi_{0}, t_{0}\right\}$, where $\varphi_{0}$ is the initial phase and $t_{0}$ is the start time of the waveform. The PN and NR waveforms are matched by minimizing the integrated squared difference, $\delta$, between them in the matching region $t_{1} \leqslant t \leqslant t_{2}$ :

$$
\delta \equiv \sum_{i=+, \times} \int_{t_{1}}^{t_{2}}\left[h_{i}^{\mathrm{PN}}(t, \boldsymbol{\mu})-a h_{i}^{\mathrm{NR}}(t, \boldsymbol{\mu})\right]^{2} \mathrm{~d} t .
$$

The minimization is carried out over the extrinsic parameters $\boldsymbol{\mu}$ of the PN waveform and an amplitude scaling factor $a,{ }^{1}$ while keeping the 'intrinsic parameters' (the two component masses) of both the PN and NR waveforms the same. The hybrid waveforms are then produced by combining the 'best-matched' PN waveforms with the NR waveforms in the following way:

$$
h_{+, \times}^{\text {hyb }}(t, \boldsymbol{\mu}) \equiv a_{0} \tau(t) h_{+, \times}^{\mathrm{NR}}(t, \boldsymbol{\mu})+(1-\tau(t)) h_{+, \times}^{\mathrm{PN}}\left(t, \boldsymbol{\mu}_{0}\right),
$$

where $\boldsymbol{\mu}_{0}$ and $a_{0}$ denote the values of $\boldsymbol{\mu}$ and $a$ for which $\delta$ is minimized, and $\tau$ is a weighting function, defined as

$$
\tau(t) \equiv\left\{\begin{array}{lll}
0 & \text { if } t<t_{1} \\
\frac{t-t_{1}}{t_{2}-t_{1}} & \text { if } \quad t_{1} \leqslant t<t_{2} \\
1 & \text { if } t_{2} \leqslant t .
\end{array}\right.
$$

It is expected that the early inspiral is better modelled by PN waveforms, as the early inspiral part of the NR waveforms is prone to larger errors. But the PN waveforms become less accurate at the late inspiral. Thus, it is important to choose a matching region where both waveforms are accurate. This also means that the hybrid waveforms constructed using two very different matching regions can potentially be quite different. If, on the other hand, we are able to show that these differences are not very significant for data-analysis purpose, this is an indication that our analysis is not heavily dependent on the choice of the matching region.

As a test of the robustness of the matching procedure, we compute the mismatch between two hybrid waveforms $h_{n}$ and $h_{n-1}$, defined as

$$
\mathcal{M M}\left(h_{n}, h_{n-1}\right) \equiv 1-\max _{t_{0}}\left[4 \operatorname{Re} \int_{0}^{\infty} \frac{h_{n}(f) h_{n-1}^{*}(f) \mathrm{e}^{\mathrm{i} 2 \pi f t_{0}} \mathrm{~d} f}{S_{h}(f)}\right],
$$

where $S_{h}(f)$ is the one-sided power spectral density of the detector noise. The subscript $n$ on the hybrid waveform $h$ means that the hybrid waveform is constructed by matching PN and NR waveforms at the $n$th cycle of the NR waveform.

The top-left panel of figure 1 shows an example set of the PN, NR and the hybrid waveforms. The hybrid waveform is constructed by matching an equal-mass NR waveform computed by the Jena group, reported in [30], with a restricted 3.5PN TaylorT1 [32] waveform. Other panels in figure 1 show the mismatch between the hybrid waveforms $h_{n}$ and $h_{n-1}$, computed using three different noise spectra. If we take $3 \%$ as the maximum allowed mismatch between hybrid waveforms $h_{n}$ and $h_{n-1}$, this preliminary exercise suggests that any matching region before $t_{1}=-150 \mathrm{M}$ is robust for constructing hybrid waveforms using the equal-mass NR waveforms considered here. This will be studied in detail in a forthcoming work [33].

1 The NR waveforms used for this work contain only the $l=2, m= \pm 2$ modes, and the PN waveforms are computed in the restricted PN approximation. Since the PN corrections to the amplitude of the PN waveforms are ignored, this will introduce an error of $\sim 8 \%$ in the amplitude of the hybrid waveforms, and hence in the horizon distances reported in figure 3; but not in the calculation of fitting factor and faithfulness. 

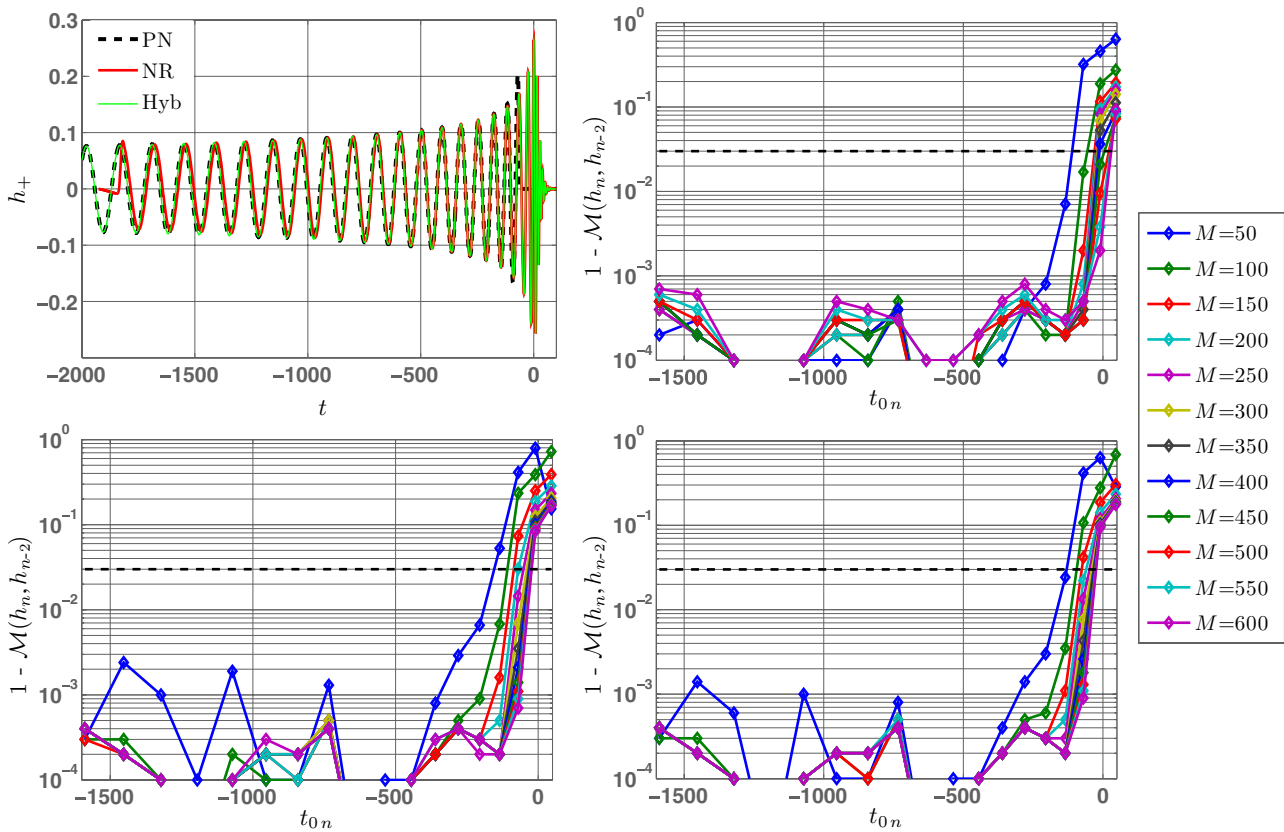

Figure 1. The top-left panel shows an example hybrid waveform (green) constructed by matching an equal-mass NR waveform (red) computed by the Jena group with a 3.5PN restricted PN waveform (black dashed). The two black vertical lines indicate the matching region $(-280 M \leqslant t \leqslant-206 M)$ employed. The rest of the panels show the mismatch between hybrid waveforms $h_{n}$ and $h_{n-1}$, where a subscript $n$ means that the hybrid waveform is constructed by matching the NR and PN waveforms at the $n$th cycle of the NR waveform. The horizontal axis reports the start time, $t_{1}$, of the $n$th cycle (in units of $M$ ). The mismatch is computed using initial LIGO (top-right), advanced LIGO (bottom-right) and Virgo (bottom-left) noise spectra. Total mass of the waveforms (in units of $M_{\odot}$ ) is shown in the legends. A mismatch of $3 \%$ is marked with a dashed horizontal line.

\section{Templates for binary black-hole coalescence}

The hybrid waveforms constructed in the previous section can be parametrized in terms of the two physical parameters of the binary, thus producing analytical waveform templates. These analytical waveforms can be used to construct template banks for matched-filter searches; thus avoiding the computational cost of generating hybrid waveforms at each grid point in the parameter space. [25] proposed a family of Fourier domain templates of the form

$$
u(f) \equiv A_{\text {eff }}(f) \mathrm{e}^{\mathrm{i} \Psi_{\text {eff }}(f)},
$$

where the effective amplitude and phase are expressed as

$$
\begin{aligned}
& A_{\text {eff }}(f) \equiv C \begin{cases}\left(f / f_{\text {merg }}\right)^{-7 / 6} & \text { if } f<f_{\text {merg }} \\
\left(f / f_{\text {merg }}\right)^{-2 / 3} & \text { if } f_{\text {merg }} \leqslant f<f_{\text {ring }} \\
w \mathcal{L}\left(f, f_{\text {ring }}, \sigma\right) & \text { if } f_{\text {ring }} \leqslant f<f_{\text {cut }},\end{cases} \\
& \Psi_{\text {eff }}(f) \equiv 2 \pi f t_{0}+\varphi_{0}+\frac{1}{\eta} \sum_{k=0}^{7}\left(x_{k} \eta^{2}+y_{k} \eta+z_{k}\right)(\pi M f)^{(k-5) / 3} .
\end{aligned}
$$


Table 1. Coefficients describing the amplitude and phase of the phenomenological waveforms. See equations (6) and (7).

\begin{tabular}{llll}
\hline Parameter & $a_{k}$ & $b_{k}$ & $c_{k}$ \\
\hline$f_{\text {merg }}$ & $6.6389 \times 10^{-1}$ & $-1.0321 \times 10^{-1}$ & $1.0979 \times 10^{-1}$ \\
$f_{\text {ring }}$ & 1.3278 & $-2.0642 \times 10^{-1}$ & $2.1957 \times 10^{-1}$ \\
$\sigma$ & 1.1383 & $-1.7700 \times 10^{-1}$ & $4.6834 \times 10^{-2}$ \\
$f_{\text {cut }}$ & 1.7086 & $-2.6592 \times 10^{-1}$ & $2.8236 \times 10^{-1}$ \\
Parameter & $x_{k}$ & & \\
$\psi_{0}$ & $-1.5829 \times 10^{-1}$ & $8.7016 \times 10^{-2}$ & $-3.3382 \times 10^{-2}$ \\
$\psi_{2}$ & $3.2967 \times 10^{1}$ & $-1.9000 \times 10^{1}$ & 2.1345 \\
$\psi_{3}$ & $-3.0849 \times 10^{2}$ & $1.8211 \times 10^{2}$ & $-2.1727 \times 10^{1}$ \\
$\psi_{4}$ & $1.1525 \times 10^{3}$ & $-7.1477 \times 10^{2}$ & $9.9692 \times 10^{1}$ \\
$\psi_{6}$ & $1.2057 \times 10^{3}$ & $-8.4233 \times 10^{2}$ & $1.8046 \times 10^{2}$ \\
$\psi_{7}$ & 0 & 0 & 0 \\
\hline
\end{tabular}

In the above expressions, $C$ is a numerical constant whose value depends on the sky-location and orientation of the binary as well as its physical parameters. For optimally located and oriented binaries, $C=\frac{M^{5 / 6} f_{\text {mrg }}^{-7 / 6}}{d \pi^{2 / 3}} \sqrt{\frac{5 \eta}{24}} . t_{0}$ is the time of arrival of the signal at the detector, $\varphi_{0}$ the initial phase, $\mathcal{L}\left(f, f_{\text {ring }}, \sigma\right) \equiv\left(\frac{1}{2 \pi}\right) \frac{\sigma}{\left(f-f_{\text {ring }}\right)^{2}+\sigma^{2} / 4}$ a Lorentzian function with width $\sigma$ centred around the frequency $f_{\text {ring }}, w$ a normalization constant chosen so as to make $A_{\text {eff }}(f)$ continuous across the 'transition' frequency $f_{\text {ring }}$, and $f_{\text {merg }}$ is the frequency at which the power law changes from $f^{-7 / 6}$ to $f^{-2 / 3}$. The phenomenological parameters $f_{\text {merg }}, f_{\text {ring }}, \sigma$ and $f_{\text {cut }}$ are written in terms of the total mass $M$ and symmetric mass ratio $\eta$ of the binary as

$$
\begin{aligned}
& \pi M f_{\text {merg }}=a_{0} \eta^{2}+b_{0} \eta+c_{0}, \\
& \pi M f_{\text {ring }}=a_{1} \eta^{2}+b_{1} \eta+c_{1}, \\
& \pi M \sigma=a_{2} \eta^{2}+b_{2} \eta+c_{2}, \\
& \pi M f_{\text {cut }}=a_{3} \eta^{2}+b_{3} \eta+c_{3} .
\end{aligned}
$$

The coefficients $a_{j}, b_{j}, c_{j}, j=0, \ldots, 3$ and $x_{k}, y_{k}, z_{k}, k=0,2,3,4,6,7$ are unique for a given family of hybrid waveforms. The coefficients corresponding to the hybrid waveforms considered here are tabulated in table 1. These are computed from seven hybrid waveforms in the range $0.25 \geqslant \eta \geqslant 0.16$ produced by matching the numerical waveforms (referred to as the 'long NR waveforms' in [25]) produced by the Jena group with restricted 3.5PN TaylorT1 waveforms.

\section{Assessing the efficiency of different searches}

The hybrid waveforms constructed in section 2 can be used to estimate the efficiency of different searches (including that proposed in the previous section) in detecting signals from binary black-hole coalescences ${ }^{2}$. Ideally, this should be done by injecting a large number of hybrid waveforms from different binaries into the detector data and by estimating the fraction of the injections detected by each search. Different search groups have already started work in this direction [34]. This section presents a simple strategy to estimate the efficiency of different

2 The obvious assumption involved is that the hybrid waveforms are sufficiently close to the signals produced by nature. This is greatly dependent on the systematic errors in the hybrid waveforms. 

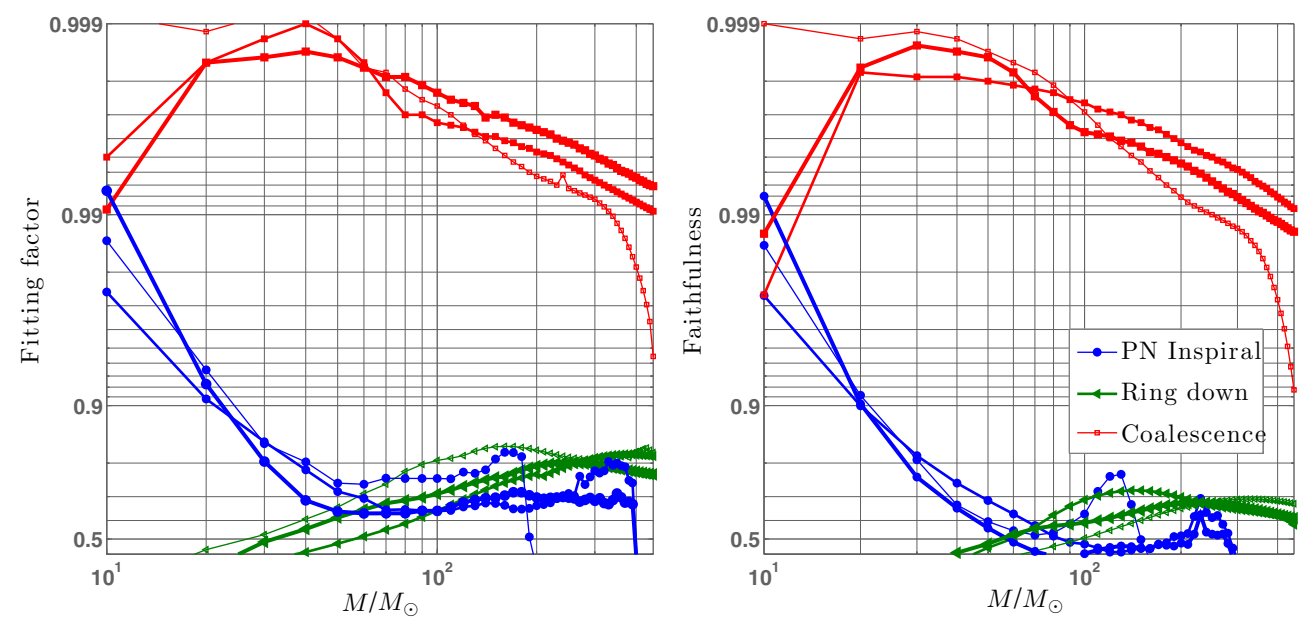

Figure 2. Fitting factors (left plots) and faithfulness (right plots) of the PN inspiral, ring-down and coalescence templates. Dots correspond to PN inspiral templates, triangles to ring-down templates, and squares to coalescence templates. The overlaps are computed using three different noise spectra-initial LIGO (thin lines), Virgo (thicker lines) and advanced LIGO (thickest lines). Horizontal axes report the total mass of the binary.

template families in detecting signals from binary black-hole coalescences. The template families being considered here are (i) restricted 3.5PN TaylorT1 inspiral templates truncated at the maximum binding-energy circular orbit [35] (ii) black-hole ring-down templates proposed in [36] and (iii) black-hole coalescence templates described in the previous section.

We compute the fitting factors [37] and faithfulness [38] of different template families with the hybrid waveforms. Fitting factor is the overlap of a template waveform with the target signal maximized over both the intrinsic $(M$ and $\eta)$ and the extrinsic $\left(t_{0}\right.$ and $\left.\varphi_{0}\right)$ parameters of the template waveform, while faithfulness is the overlap maximized over only the extrinsic parameters of the template. Faithfulness is a measure of how good the template waveform is in both detecting a signal and estimating its parameters. However, the fitting factor is aimed at finding whether or not a template family is good enough in detecting a signal without reference to its use in estimating the parameters.

The fitting factors and faithfulness of three different template families, using the hybrid waveforms as target signals, are plotted in figure 2. Maximization over the intrinsic parameters is performed with the aid of the Nelder-Mead downhill simplex algorithm. Low frequency cutoff is chosen to be equal to $40 \mathrm{~Hz}$ for initial LIGO and $20 \mathrm{~Hz}$ for Virgo and advanced LIGO. As expected, PN inspiral templates produce very good overlaps with the target signals in the low-mass regime (where inspiral is the dominant part), and ring-down templates produce good overlaps in the high-mass regime (where ring down is the dominant part). The black-hole coalescence templates continue to produce very good overlaps over the entire mass range. Note that, if we assume homogeneous and isotropic distribution of sources, the fraction of sources detectable by a template family is proportional to the cube of the fitting factor [39]. The 'event loss' due to mismatch between the template and the true signal is larger than the canonical $10 \%$ when the fitting factor is less than $0.965 .{ }^{3}$ This figure suggests that the template

3 It should be noted that the fitting factor is not the only consideration in an actual search strategy. Other factors such as computational cost and false alarm rate also play a decisive role in the choice of a template family. However such a detailed study is beyond the scope of this paper. 

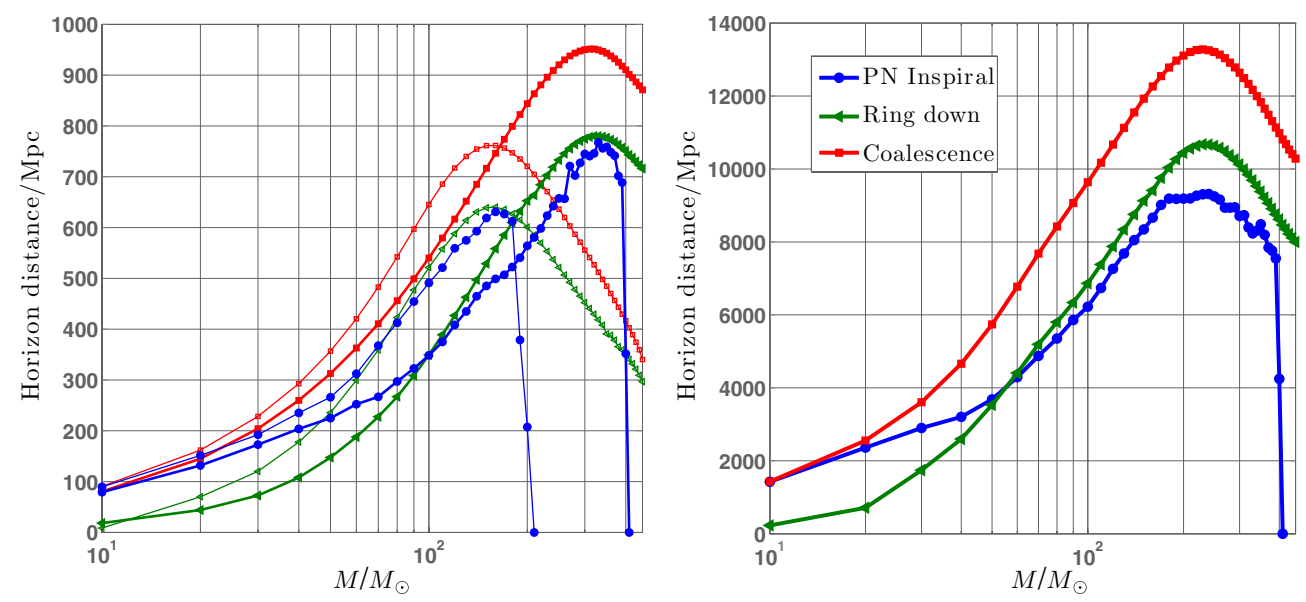

Figure 3. The left plots show the horizon distances of PN inspiral, ring-down and coalescence templates in the case of initial LIGO (thin lines) and Virgo (thick lines) noise spectra. The right plots show the same for advanced LIGO. Dots correspond to PN inspiral templates, triangles to ring-down templates, and squares to coalescence templates. Horizontal axes report the total mass of the binary, and the vertical axes report the effective distance to optimally oriented equal-mass binaries producing a sub-optimal SNR of 8 at the detector output. The sharp drop in the PN horizon distance is a result of the (different) lower cutoff frequencies of the detectors.

family proposed in section 3 can be used to search for binary black-hole coalescences over almost the entire mass range considered here losing not more than $10 \%$ of the events that are detectable by optimal filtering. Binaries with total mass $\lesssim 15 M_{\odot}$ are detectable using PN inspiral templates with $<10 \%$ event loss, while the binaries in the mass range considered here cannot be detected with $<10 \%$ event loss using black-hole ring-down templates ${ }^{4}$. Faithfulness of the coalescence templates is also almost always greater than 0.965 (comparable to the fitting factors), while that of the other templates is considerably smaller in general. This also means that the parameters estimated by the PN and ring-down templates will be biased significantly. This will be studied in detail in a forthcoming work [33].

We can also calculate the 'distance reach' of these searches. Since the template waveforms described in equations (5) and (6) are shown to be very close (fitting factors $>0.95$ ) to the hybrid waveforms, the effective distance $d_{\text {opt }}$ to binaries producing a certain optimal signalto-noise ratio (SNR) $\rho$ at the detector can be computed analytically using these template waveforms, as

$$
d_{\mathrm{opt}}=\frac{2}{\rho}\left[\int_{f_{\mathrm{low}}}^{f_{\mathrm{cut}}} \frac{A_{\mathrm{eff}}(f) \mathrm{d} f}{S_{h}(f)}\right]^{1 / 2} .
$$

Since the fitting factor (FF) is the fraction of the optimal SNR that can be achieved using a sub-optimal filter, the effective distance $d_{\text {subopt }}$ to the (optimally oriented) binaries producing a sub-optimal SNR $\rho$ by a template family is given by $d_{\text {subopt }}=d_{\text {opt }}$ FF. Figure 3 compares the effective distance to optimally located and oriented binaries producing a sub-optimal SNR of 8 at the detector output using the three different template families discussed above. For the black-hole coalescence templates, the horizon distance reaches peak values of around $760 \mathrm{Mpc}\left(\right.$ at $\left.150 M_{\odot}\right), 950 \mathrm{Mpc}\left(325 M_{\odot}\right)$ and $13.3 \mathrm{Gpc}\left(225 M_{\odot}\right)$ for initial LIGO, Virgo

4 Note that the overlaps are maximized over the initial phase $\varphi_{0}$ of the ring down also, unlike what is proposed in [36]. 
and advanced LIGO, respectively. For PN inspiral templates, the peak values are $630 \mathrm{Mpc}$ $\left(160 M_{\odot}\right), 770 \mathrm{Mpc}\left(325 M_{\odot}\right)$ and $9.2 \mathrm{Gpc}\left(250 M_{\odot}\right)$, while for the ring-down templates, the corresponding values are $640 \mathrm{Mpc}\left(150 M_{\odot}\right), 780 \mathrm{Mpc}\left(325 M_{\odot}\right)$ and $10.6 \mathrm{Gpc}\left(225 M_{\odot}\right)$. It may be noted that, since the event rate is proportional to the cube of the distance reach, a $20 \%$ loss in the distance reach means a $50 \%$ loss in the event rate.

\section{Summary and future work}

Recent progress in the theoretical modelling of coalescing binary black holes has important applications in the search for gravitational waves from binary black-hole coalescences. 'Complete' gravitational waveforms can be constructed by combining results from analytical and numerical calculations. These waveforms can be parametrized to produce analytical waveform templates which can be used to densely cover the parameter space of the binary that will be searched over by matched-filtering techniques. This template family will allow us to coherently search over all the three stages of the binary. The advantages of this 'coherent search' include improved SNR, and hence improved distance reach for the search, potential reduction of the false-alarm rate and improved parameter estimation. The application of the complete waveforms is not limited to template-based searches. For example, these waveforms can also be used in the burst searches, customized for detection of signals from coalescing black-hole binaries. In this case, a small bank of representative waveforms can be used to survey the parameter space of the binary [40]. The complete waveforms can also be used to estimate the efficiency of different search methods. A preliminary comparison of three different template-based searches is presented in this paper. More robust ways of comparing the efficiency of different searches is an ongoing effort [34].

\section{Acknowledgments}

I would like to thank the numerical relativity groups of Albert Einstein Institute and University of Jena for sharing their results of binary black-hole simulations, and all the members of the AEI-Jena NRDA Collaboration for very useful discussions. Conversations with Yanbei Chen, Sascha Husa, Mark Hannam, Badri Krishnan, Sergey Klimenko and Stas Babak were particularly valuable.

\section{References}

[1] Blanchet L et al 2004 Phys. Rev. Lett. 93091101 (Preprint gr-qc/0406012)

[2] Arun K G et al 2004 Class. Quantum Grav. 213771 (Preprint gr-qc/0404185)

[3] Teukolsky S and Press W 1974 Astrophys. J. 193 443-61

[4] Pretorius F 2005 Phys. Rev. Lett. 95121101 (Preprint gr-qc/0507014)

[5] Campanelli M et al 2006 Phys. Rev. Lett. 96111101 (Preprint gr-qc/0511048)

[6] Baker J G et al 2006 Phys. Rev. Lett. 96111102 (Preprint gr-qc/0511103)

[7] Baker J G et al 2006 Astrophys. J. 653 L93-6 (Preprint astro-ph/0603204)

[8] González J A et al 2007 Phys. Rev. Lett. 98091101 (Preprint gr-qc/0610154)

[9] Campanelli M, Lousto C O and Zlochower Y 2006 Phys. Rev. D 74041501 (Preprint gr-qc/0604012)

[10] Campanelli M, Lousto C O and Zlochower Y 2006 Phys. Rev. D 74084023 (Preprint gr-qc/0608275)

[11] Herrmann F, Hinder I, Shoemaker D, Laguna P and Matzner R A 2007 Preprint gr-qc/0701143

[12] Koppitz M et al 2007 Phys. Rev. Lett. 99041102 (Preprint gr-qc/0701163)

[13] González J A et al 2007 Phys. Rev. Lett. 98231101 (Preprint gr-qc/0702052)

[14] Campanelli M et al 2007 Astrophys. J. 659 L5-8 (Preprint gr-qc/0701164)

[15] Campanelli M et al 2007 Phys. Rev. D 75064030 (Preprint gr-qc/0612076)

[16] Pollney D et al 2007 Phys. Rev. D 76124002 (Preprint 0707.2559) 
[17] Rezzolla L et al 2007 Preprint 0708.3999

[18] Abbott B et al (LIGO Scientific Collaboration) 2008 Phys. Rev. D 77062002 (Preprint 0704.3368)

[19] Goggin L M (LIGO Scientific Collaboration) 2006 Class. Quantum Grav. 23 S709-13

[20] Schutz B F 1986 Nature (London) 323310

[21] Holz D E and Hughes S A 2005 Astrophys. J. 62915 (Preprint astro-ph/0504616)

[22] Damour T and Nagar A 2007 Phys. Rev. D 76064028 (Preprint 0705.2519)

[23] Buonanno A et al 2007 Phys. Rev. D 76104049 (Preprint 0706.3732)

[24] Pan Y et al 2008 Phys. Rev. D 77024014 (Preprint 0704.1964)

[25] Ajith P et al 2007 Preprint 0710.2335 (to appear in Phys. Rev. D)

[26] Ajith P et al 2007 Class. Quantum Grav. 24 S689-99 (Preprint 0704.3764)

[27] Baker J G, van Meter J R, McWilliams S T, Centrella J and Kelly B J 2007 Phys. Rev. Lett. 99181101 (Preprint gr-qc/0612024)

[28] Buonanno A, Cook G B and Pretorius F 2007 Phys. Rev. D 75124018 (Preprint gr-qc/0610122)

[29] Berti E et al 2007 Phys. Rev. D 76064034 (Preprint gr-qc/0703053)

[30] Hannam M et al 2008 Phys. Rev. D 77044020 (Preprint 0706.1305)

[31] Boyle M et al 2007 Phys. Rev. D 76124038 (Preprint 0710.0158)

[32] Damour T et al 2001 Phys. Rev. D 63044023 (Preprint gr-qc/0010009)

[33] Ajith $\mathrm{P}$ et al 2007 in preparation

[34] Santamaria L, Fairhurst S and Krishnan B 2007 Private communication

[35] Blanchet L 2002 Phys. Rev. D 65124009

[36] Creighton J D E 1999 Phys. Rev. D 60022001 (Preprint gr-qc/9901084)

[37] Apostolatos T A 1995 Phys. Rev. D 52 605-20

[38] Damour T, Iyer B R and Sathyaprakash B S 1998 Phys. Rev. D 57 885-907

[39] Owen B J 1996 Phys. Rev. D 53 6749-6761 (Preprint gr-qc/9511032)

[40] Klimenko S 2007 Private communication 